\title{
Design of Transistor Regulated Power Supplies*
}

\author{
R. D. MIDDLEBROOKं †, MEMBER, IRE
}

\begin{abstract}
Summary-A new form of transistor series regulated power supply is presented which permits unusually good performance characteristics to be realized with simple and economical circuitry. Expressions are given for the open-circuit output voltage and the output resistance in terms of the supply voltages and the circuit parameters. Application of the new circuit to two practical laboratory regulated power supplies is described, the specifications of which are: 0.5 ampere at 18 to 22 volts, and 1 a at 5 to $25 \mathrm{v}$. The output resistance is of the order of $0.01 \mathrm{ohm}$ for both supplies; for sudden change of load current between minimum and maximum the transient in output voltage is $80 \mathrm{mv}$ or less, and decays in $40 \mu \mathrm{sec}$ or less. At full load, ripple in the output voltage is less than $5 \mathrm{mv}$ and line voltage variations of \pm 10 per cent produce output variations of less than $5 \mathrm{mv}$. Each supply uses only one line transformer.
\end{abstract}

\section{INTRODUCTION}

$\prod$ HE DESIGN of vacuum tube regulated power supplies is well-established. Such supplies are suited for powering equipment in which relatively low currents at high voltages are required. However, for high-current low-voltage applications, vacuum tube supplies become bulky and inefficient, and the design is conveniently implemented with transistors instead of with tubes. Although various types of transistor regulated power supplies have been described ${ }^{1-5}$, most of these possess comparatively low current ratings and poor transient response unless rather complex circuitry is used.

This paper presents a new form of transistor series regulated power supply which permits unusually good performance characteristics to be realized with simple and economical circuitry. Expressions are given for the regulated output voltage and the output resistance in terms of the supply voltages and circuit parameters.

Practical circuits for two laboratory regulated power supplies are presented. The input in each case is $110 \mathrm{v}$ $60 \mathrm{cps}$ and the other specifications are as follows:

Supply No. $1: 0.5$ a at 18 to $22 \mathrm{v}$, output resistance $0.03 \mathrm{ohm}$.

Supply No. 2: 1 a at 5 to $25 \mathrm{v}$, output resistance $<0.01 \mathrm{ohm}$.

* Original manuscript received by the IRE, February 4, 1957; revised manuscript received, July 15,1957 . The work described in this paper was performed for ElectroData Corp., Div. of Burroughs, Pasadena, Calif., and is reported by kind permission of ElectroData Corp.

$\dagger$ Elec. Eng. Dept., Calif. Inst. Tech., Pasadena, Calif.

${ }^{1}$ S. Sherr and P. M. Levy, "Design consideration for semiconductor regulated power supplies," Electronic Design, vol. 4, pp. 2225 ; July 15, 1956.

2 F. H. Chase, "Power regulation by semiconductors," Elec. Eng., vol. 75, pp. 818-822; September, 1956.

3 J. W. Keller, "Regulated transistor power supply design," Electronics, vol. 29, pp. 168-171; November, 1956.

${ }^{4}$ M. Lillienstein, "Transistorized regulated power supply," Electronics, vol. 29, pp. 169-171; December, 1956.

${ }^{5}$ L. P. Hunter, "Handbook of Semiconductor Electronics," McGraw-Hill Book Company, Inc., New York, N. Y., pp. 13.26-13.28; 1956.
The transient response of each supply is excellent: sudden increase of load current from zero to maximum produces a transient in output voltage of less than $80 \mathrm{mv}$ with a recovery time of about $10 \mu \mathrm{sec}$; sudden decrease of load current from maximum to zero produces a transient in output voltage of less than $40 \mathrm{mv}$ with a recovery time of about $40 \mu \mathrm{sec}$. At full load, ripple in the output voltage is less than $5 \mathrm{mv}$ and line voltage variations of \pm 10 per cent produce output voltage variations of less than $5 \mathrm{mv}$. Each supply uses only one line transformer.

\section{Qualitative Development of the New Series Regulator Circuit}

A simple form of standard series regulated power supply using $p-n-p$ transistors is shown in Fig. 1 . The voltage $E$ is the regulated output and $E_{p}$ is the unregulated "raw" input voltage with internal resistance $R_{p}$. It is required that the voltage $E$ should be as constant as possible with respect to load current $I$ for both steady-state and transient conditions, and should be as independent as possible of input voltage and circuit parameter variations.

If in Fig. 1, the base current of the transistor $T 4$ is neglected compared to the current through the potential divider, and if the base current of transistor $T 1$ is neglected compared to the collector current of $T 4$, an equation for the output voltage may be simply derived as follows. In the steady state the output voltage $E$ is given by

$$
E=n(e+v)
$$

where

$e=$ the reference voltage developed across the zener diode $D 1$,

$v=$ the emitter-to-base dc voltage of $T 4$ (approximately a tenth of a volt), and

$n=$ ratio of the potential divider as defined in Fig.1.

At any given load current $I$, there will be a voltage drop between the emitter and base of $T 1$ which is approximately $R_{e} I$, where $R_{e}$ is the effective emitter-to-base resistance of $T 1$. The voltage $v$ at the input of $T 4$ adjusts itself so that the collector current of $T 1$ produces a voltage drop $V$ across resistance $R$ so that

$$
V=E_{p}-R_{p} I-E-R_{e} I .
$$

Combining (1) and (2) gives

$$
E=\left(n e+\frac{E_{p}}{G}\right)-\left(\frac{R_{p}+R_{e}}{G}\right) I
$$




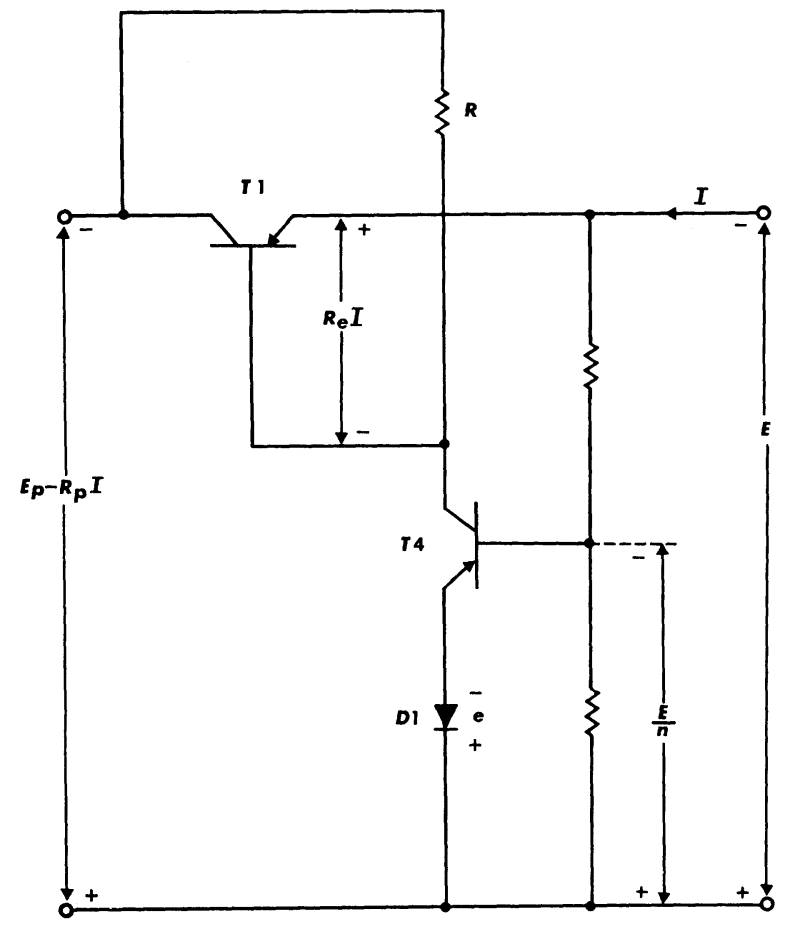

Fig. 1-Simple series regulator circuit, in which $E$ is the regulated output voltage, and the supply voltage is $E_{p}$ with internal resistance $R_{p}$. The output resistance is approximately $\left(R_{\boldsymbol{e}}+R_{p}\right) / G$.

where $G=V / n v$, and is the dc gain of $T 4$ and the potential divider. It is assumed that $G \gg 1$ in the derivation of (3).

In an ideal regulator $E=n e$, and thus (3) indicates the extent to which the circuit of Fig. 1 fails to be ideal. The undesired terms in (3) show that a practical regulator exhibits a finite output resistance and that the opencircuit output voltage is partially dependent on the raw supply voltage. In a typical case, suppose that the power supply is to provide zero to 1 a at $20 \mathrm{v}$ and let $e=5 \mathrm{v}, R_{e}=1 \mathrm{ohm}, R_{p}=9 \mathrm{ohm}$, and $G=10$. At maximum load of $1 \mathrm{a}, 9 \mathrm{v}$ will be dropped in the internal resistance $R_{p}$ of the raw supply, so in order to maintain at least $1 \mathrm{v}$ reverse bias between the base and collector of $T 1$, the supply voltage $E_{p}$ must be at least $30 \mathrm{v}$. To provide $E=20 \mathrm{v}$ with $e=5 \mathrm{v}$ requires $n=4$ if the regulator approaches ideal performance. Substitution of the given figures into the undesired terms of (2) shows that $E_{p} / G=3 \mathrm{v},\left(R_{p}+R_{e}\right) / G=1 \mathrm{v}$ at 1 a load current. Thus if there is any variation in $E_{p}$ (such as change in supply voltage or ripple) the variation in the output voltage $E$ is $3 / 20$ of the variation in $E_{p}$. The factor $\left(R_{p}+R_{e}\right) / G=1$ ohm is the output resistance $R_{0}$ of the regulated supply, which is an improvement by about a factor of 10 over the output resistance $R_{p}$ of the raw supply.

However, an output resistance of $1 \mathrm{ohm}$ is still too high for many applications. A circuit modification which will further reduce the output resistance presents itself after a qualitative examination of how the circuit of Fig. 1 operates when the load current $I$ is changed.

In the circuit of Fig. 1, an increase in load current $I$ tends to cause a drop in output voltage $E$ not only be-

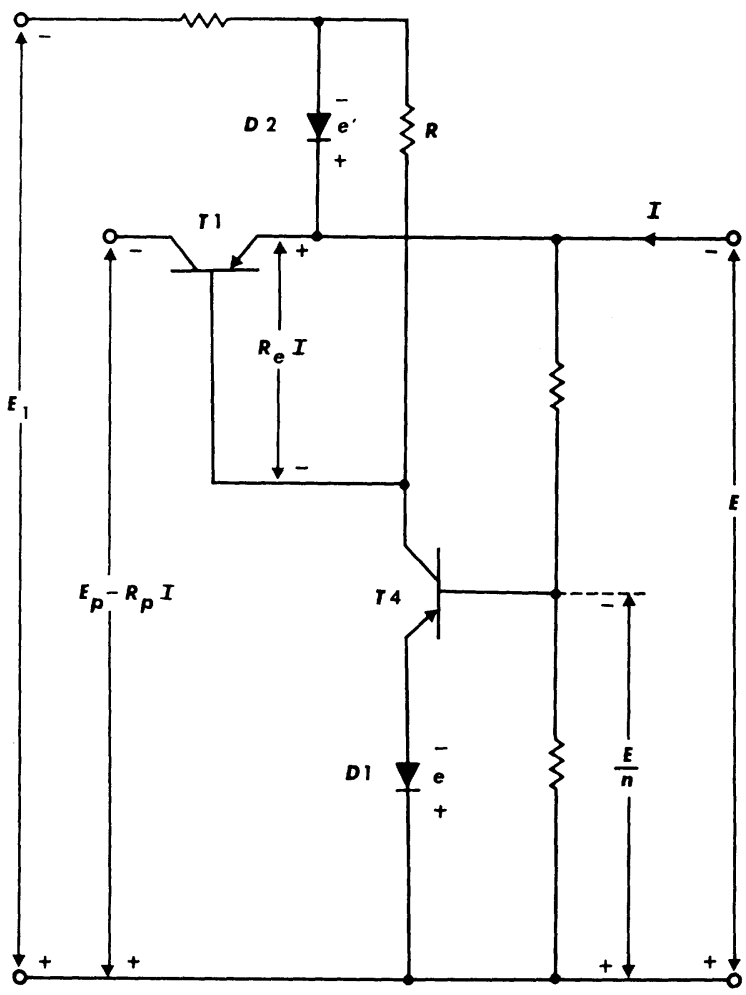

Fig. 2-Principle of the new series regulator circuit, in which the output resistance is reduced approximately to $R_{e} / G$.

cause of the larger drop $R_{e} I$ in $T 1$, but also because of the larger drop $R_{p} I$ in the internal resistance of the raw supply $E_{p}$ which is connected to the output via the resistor $R$ and transistor $T 1$. Thus the amplifier $T 4$ is required to compensate for both these drops, and hence the factor $\left(R_{p}+R_{e}\right)$ occurs in (3). Obviously, if some means could be found to maintain the voltage at the top end of $R$ constant with respect to changes in load, the output resistance would be equal to $R_{e} / G$ instead of to $\left(R_{p}+R_{e}\right) / G$, or $0.1 \mathrm{ohm}$ instead of $1 \mathrm{ohm}$ for the above numerical values. This may be accomplished by the circuit of Fig. 2, in which the top end of $R$ is connected to a voltage more negative than the output voltage by a fixed amount. The presence of the zener diode $D 2$ insures that the voltage at the top end of $R$ is always essentially constant for all load currents, and thus the above conditions for lower output resistance are realized.

An expression for the output voltage $E$ in the circuit of Fig. 2 is easily obtained by substituting $E+e^{\prime}$ for $E_{p}-R_{p} I$ in (3), which leads to

$$
E=n e+\frac{e^{\prime}}{G}-\frac{R_{e}}{G} I .
$$

It is seen that the output resistance $R_{0}$ is indeed $R_{e} / G$, or $0.1 \mathrm{ohm}$ for the figures given previously. Further, the output voltage is independent of $E_{p}$ and the auxiliary supply $E_{1}$, and thus $E$ will be independent of supply voltage variations or ripple.

It is apparent that the circuit of Fig. 2 provides a marked improvement over that of Fig. 1, and yet re- 
quires only two more components and an extra (lowcurrent) supply voltage. The simple semiquantitative discussion above is sufficient to explain the principle of the modified regulated supply circuit of Fig. 2, but in practical circuits several additional factors must be taken into account. The most important of these are that the zener reference diodes $D 1$ and $D 2$ possess finite dynamic resistance (thus the voltages $e$ and $e^{\prime}$ are not quite constant for different currents), and that for maximum load currents in excess of a few milliamperes the base current of $T 1$ will certainly not be negligible compared to the collector current of $T 4$. All these factors affect the gain $G$ of $T 4$, and also cause the output voltage not to be completely independent of the supply voltage. A more detailed analysis of this new circuit follows in the next section.

\section{Analysis of the Regulator Circuit}

A practical embodiment of the principle described in the previous section is shown in Fig. 3. The most important change is the addition of two more transistors $T 2$ and $T 3$, connected as emitter followers, to provide current amplification so that load currents of several amperes may be drawn, and yet to keep the base current of $T 3$ small enough to be comparable with or smaller than the collector current of $T 4$. A base current in $T 3$, which is not negligible compared to the collector current of $T 4$, means that the input resistance of $T 3$ shunts appreciably the resistance $R$. The three transistors $T 1, T 2$, and $T 3$ will usually be adequate for load currents up to 1 a. An ammeter will normally be required to monitor the load current, and must be inserted inside the feedback loop; otherwise, the output resistance of the supply will be increased by the resistance of the meter.

If the dc current through the transistor T4 is insufficient to maintain the reference diode $D 1$ in the breakdown region, the auxiliary supply $E_{2}$ may be necessary to provide extra current. It is desirable that $E_{2}$ be obtained from the same line transformer that supplies the external load current, in which case $E_{2}$ will contain a ripple component and will vary with load current $I$ owing to the imperfect regulation of the raw supply. This effect may be accounted for by supposing the supply $E_{2}$ to contain a "transfer internal resistance" $R_{2}$, which may be of the order of $10 \mathrm{ohms}$. The other auxiliary supply $E_{1}$ will possess in a similar way a transfer internal resistance $R_{1}$.

The resistance $R_{f}$ is very small, of the order of a fraction of an ohm, and is inserted to provide positive feedback proportional to the load current so that the output resistance of the regulated supply can be made zero or slightly negative. The feedback resistance may not always be necessary, but will be included in the analysis so that its effect can be evaluated.

Analysis of the circuit of Fig. 3 requires finding the equation corresponding to (4) for the regulated output voltage, and is conveniently broken down into two

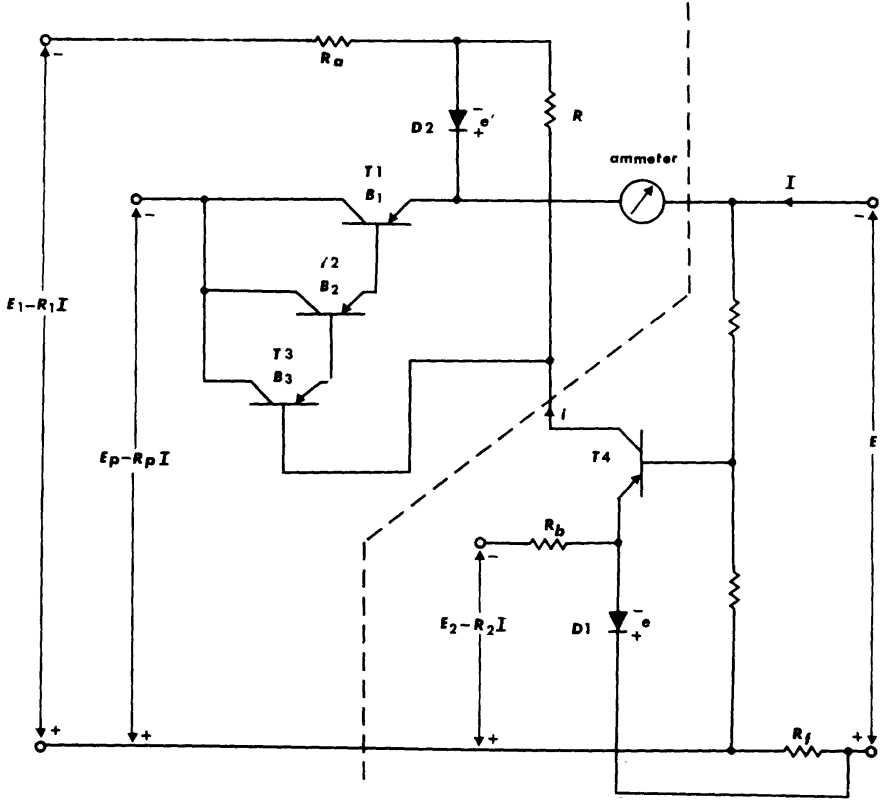

Fig. 3-Practical embodiment of the circuit principle of Fig. 2.

parts. First, an expression for the collector current $i$ of transistor $T 2$ is found in terms of the output voltage $E$ by consideration of the voltage amplifier part of the circuit to the right of the dashed line in Fig. 3. Second, an expression for $E$ in terms of $i$ is obtained by consideration of the current amplifier part of the circuit to the left of the dashed line in Fig. 3. Elimination of $i$ from the two resulting equations leads to the desired expression for the output voltage $E$ :

$$
\begin{aligned}
E= & {\left[n e+\frac{r}{R_{b}} n E_{2}+\frac{r^{\prime}}{R_{a}} \frac{1}{G} E_{1}+\frac{1}{G} e^{\prime}\right] } \\
& -I\left[\frac{\left(R_{e 1}+R_{e 2}+R_{e 3}\right)+R / B_{1} B_{2} B_{3}}{G}\right. \\
& \left.\quad+\frac{R_{1}+R_{m}}{\left(R_{a} / r^{\prime}\right) G}+\frac{r}{R_{b}} n R_{2}-\frac{1+n}{n} R_{f}\right]
\end{aligned}
$$

where

$$
\begin{aligned}
e, r= & \text { the reference voltage, dynamic resist- } \\
& \text { ance of zener diode } D 1, \\
e^{\prime}, r^{\prime}= & \text { the corresponding quantities of zener } \\
& \text { diode } D 2, \\
R_{m}= & \text { the resistance of the ammeter, } \\
B_{1}, B_{2}, B_{3}= & \text { the common-emitter dc current gains of } \\
& T 1, T 2, T 3, \\
R_{e 1}, R_{e 2}, R_{e 3}= & \text { effective emitter-base resistances of } T 1, \\
& T 2, T 3 \text { (defined in each case as the } \\
& \text { ratio: change in emitter-base voltage/ } \\
& \text { change in load current } I \text { ), } \\
n= & \text { the ratio of the potential divider, de- } \\
& \text { fined as in Fig. } 1, \\
G= & \text { is the dc gain of the potential divider } \\
& \text { and the amplifier } T 4,
\end{aligned}
$$

and the other quantities are as shown in Fig. 3. The gain $G$ corresponds to the quantity $V / n v$ previously 
mentioned, and is given by

$$
G=R / n\left[r_{e}+r+\left(r_{p}+r_{b}\right) / B_{4}\right]
$$

where $r_{p}$ is the resistance of the two potential divider resistors in parallel, and $r_{e}, r_{b}$, and $B_{4}$ are the emitter resistance, base resistance, and common-emitter dc current gain of $T 4$.

In the derivation of (5), the following good approximations are made:

$$
\begin{aligned}
R_{b} & \gg r \\
R_{a} & \gg r^{\prime} \\
R & \gg r^{\prime} \\
\left(R_{a} / r^{\prime}\right) G & \gg 1 .
\end{aligned}
$$$$
B \gg 1 \text { for all transistors }
$$

It is further assumed that the load current $I$ is considerably larger than the current drain through the potential divider, and is also considerably larger than the current through the diode $D 2$.

Eq. (5) may be written

$$
E=E_{0}-R_{0} I
$$

where $E_{0}$ is the open-circuit output voltage and $R_{0}$ is the effective output resistance of the regulated supply. The criteria of a satisfactory design are that $E_{0}$ should be as independent as possible of the raw supply voltages $E_{p}, E_{1}$, and $E_{2}$, and the output resistance should be as small as possible (less than $1 \mathrm{ohm}$ ). Eq. (5) shows the relative effects of the raw supply voltages and the various internal resistances which cause the output voltage to deviate from the ideal value $E=n e$. A discussion of the implications of (5) is desirable, and is given in the next section.

\section{Discussion of the Analytical Results}

Consider first the effects of the raw supply voltages on the open-circuit output voltage $E_{0}$, where, from (5) and (12),

$$
E_{0}=n e+\frac{r}{R_{b}} n E_{2}+\frac{r^{\prime}}{R_{a}} \frac{1}{G} E_{1}+\frac{1}{G} e^{\prime}
$$

In an ideal regulator $E_{0}=n e$, and thus the other terms in (13) are undesirable and should be minimized in a good design. In order to obtain a high value of the ratio $R_{a} / r^{\prime}$, the supply $E_{1}$ should be at least twice the output voltage $E$. Let typical figures again be taken of $e=5 \mathrm{v}$, $n=4$; then $E_{0}$ will be equal to $20 \mathrm{v}$ plus correction terms. The supply $E_{1}$ should be at least $40 \mathrm{v}$, and for $G=10$ and $R_{a} / r^{\prime}=100$ the term in $E_{1}$ is equal to $0.04 \mathrm{v}$, or 0.2 per cent of the output voltage. Thus $E_{0}$ is almost completely independent of $E_{1}$, and practically none of any ripple on $E_{1}$ will get through to the output.

In some cases the supply $E_{2}$ will not be required at all, since sufficient dc current to break down the reference diode $D 1$ may be available through transistor $T 4$. In this case, of course, the terms in $E_{2}$ will not appear in the equation for $E_{0}$. However, if extra current is required for the diode $D 1$, it may be obtained from the supply $E_{p}$ in which case $E_{2}=E_{p}$. For $E_{p}=30 \mathrm{v}$, the ratio $R_{b} / r$ may be about 100 , and so the term in $E_{2}$ in (13) will be equal to $1.2 \mathrm{v}$. This represents 6 per cent of the output voltage $E_{0}$, a figure which is undesirably high since variations in line voltage and ripple will thus be transmitted to the regulated output with appreciable magnitude. A way to overcome this problem is to make $E_{2}$ equal not to $E_{p}$ but to $E$; that is, to derive the extra current for $D 2$ from the regulated output instead of from the raw input. Putting $E_{2}=E$ in (5) leads to

$$
E_{0}=\frac{1}{1-n r / R_{b}}\left(n e+\frac{r^{\prime}}{R_{a}} \frac{1}{G} E_{1}+\frac{1}{G} e^{\prime}\right)
$$

and the result is essentially the same as when $E_{2}=0$ except that the no-load output voltage is increased by the factor $1 /\left(1-n r / R_{b}\right)$ which, however, is close to unity.

The remaining term to consider in (5) is that in $e^{\prime}$. If $e^{\prime}=5 \mathrm{v}$, this term amounts to 2 per cent of the output voltage and is not detrimental since $e^{\prime}$ is itself a reference voltage.

To summarize the above discussion of the open-circuit output voltage, the typical numerical values obtained above are shown below in a form in which the terms correspond to those in (14), where $E_{2}=E$ :

$$
\begin{gathered}
E_{0}=\frac{1}{1-0.04}(20+0.04+0.5) \\
E_{0}=21.36 \mathrm{v} .
\end{gathered}
$$

Consider next the effects of the various internal resistances on the output resistance $R_{0}$. From (5) and (12),

$$
\begin{aligned}
R_{0}= & \frac{\left(R_{e 1}+R_{e 2}+R_{e 3}\right)+R / B_{1} B_{2} B_{3}}{G} \\
& +\frac{R_{1}+R_{m}}{\left(R_{a} / r^{\prime}\right) G}+\frac{r}{R_{b}} n R_{2}-\frac{1+n}{n} R_{f} .
\end{aligned}
$$

In an ideal regulator $R_{0}$ should be as small as possible, and hence all the terms in (17) should be minimized in a good design. Each term which contributes to the output resistance $R_{0}$ can be identified with an independent process occurring in the circuit of Fig. 3.

The quantity $\left(R_{e 1}+R_{e 2}+R_{e 3}\right)$ is the total effective internal resistance of the current-amplifying transistors $T 1, T 2$, and $T 3$ and corresponds to the internal resistance $R_{e}$ of the single transistor shown in the circuit of Fig. 2. As predicted by the simple analysis given in connection with Fig. 2, the internal resistance of the current-amplifying transistor contributes a term $\left(R_{e 1}+R_{e 3}\right.$ $\left.+R_{e 3}\right) / G$ to the output resistance of the supply. However, in the simple analysis it was assumed that the base current of the current-amplifying transistor was negligible compared to the current through $R$, that is, the resistance looking into the base of the current-amplifying transistor was much larger than the resistance $R$. In the 
more accurate analysis given above this assumption was not made, and the presence of this shunting effect is represented by the term in $R$ in (17).

Some typical figures again will help to give a feeling for the relative magnitudes of the terms in (17) for the output resistance. Suppose that $G=10, R=6000 \mathrm{ohms}$, $B_{1}, B_{2}, B_{3}=30,40,50$, and $R_{e 1}, R_{e 2}, R_{e 3}=0.70,0.15,0.15$ ohms respectively. The total effective internal resistance $R_{e}$ of transistors $T 1, T 2$, and $T 3$ is then $1 \mathrm{ohm}$, and the additional resistance contributed by the term in $R$ is 0.1 $\mathrm{ohm}$. Hence, the component of output resistance arising from these two sources is $(1+0.1) / 10=0.11 \mathrm{ohm}$, which may be compared with the result of $0.10 \mathrm{ohm}$ which would be calculated by neglecting the shunting effect.

From (17) it is seen that an additional component of output resistance arises from the presence of the ammeter resistance $R_{m}$, and of the auxiliary supply transfer internal resistance $R_{1}$. Typical values are $R_{m}=1 \mathrm{ohm}$, $R_{1}=10 \mathrm{ohm}$. However, it is seen that the resulting component of output resistance is the sum of the two resistances divided not by $G$ alone, but by $\left(R_{a} / r^{\prime}\right) G$. For the above typical figures, this component of output resistance is $0.011 \mathrm{ohm}$, and is only 10 per cent of the component due to $R_{e}$ and $R$.

A third component of output resistance is introduced by the transfer internal resistance $R_{2}$ of the auxiliary supply $E_{2}$. As described in connection with the opencircuit output voltage $E_{0}$, the supply $E_{2}$ will not be required in some cases, and so $R_{2}=0$. However, if $E_{2}$ is taken from the main supply voltage $E_{p}, R_{2}=R_{p}$ and may be about $10 \mathrm{ohm}$. For $R_{b} / r=100, n=4$ as before, the component of output resistance due to $R_{2}$ will be $0.4 \mathrm{ohm}$ and is undesirably large. This problem may be resolved by again deriving the extra current supply for diode $D 1$ from the output voltage $E$ instead of from $E_{p}$. In this case $R_{2}=0$, and putting $E_{2}=E$ in (5) gives

$$
\begin{array}{r}
R_{0}=\frac{1}{1-n r / R_{b}}\left[\frac{\left(R_{e 1}+R_{e 2}+R_{e 3}\right)+R / B_{1} B_{2} B_{3}}{G}\right. \\
\left.+\frac{R_{1}+R_{m}}{\left(R_{a} / r^{\prime}\right) G}-\frac{1+n}{n} R_{f}\right]
\end{array}
$$

and the result is essentially the same as when $E_{2}$ and $R_{2}$ are zero, except that the output resistance is increased by the factor $1 /\left(1-n r / R_{b}\right)$ which, however, is close to unity.

The remaining term to consider in (17) is that due to the feedback resistance $R_{f}$. As described in Section III, $R_{f}$ introduces positive current feedback whose magnitude can be adjusted to make $R_{0}$ zero or even negative. From (18) the value of $R_{f}$ which would be required to make $R_{0}=0$ for the above typical figures, and where $E_{2}=E_{p}$ and $R_{2}=R_{p}$, is given by

$$
0=0.11+0.011+0.4-(5 / 4) R_{f}
$$

or

$$
R_{f}=0.417 \mathrm{ohm}
$$

A resistance of this small magnitude is furnished by a few inches of wire, and, in practice, the best way to obtain the desired value of $R_{f}$ is to adjust the length of a piece of wire in the appropriate place in the completed regulated supply circuit until the output resistance is zero. This method of reducing the output resistance is not particularly satisfactory, however, since the adjustment is awkward to make and in any case will only be correct for a particular value of $n$. Thus, if a variable output voltage $E$ is required (which is obtained by varying the potential divider ratio $n$ ), the output resistance $R_{0}$ may vary considerably over the range of output voltage. Therefore, it is preferable to design for a low inherent output resistance by making $G$ as large as possible.

To summarize the above discussion of the output resistance, the typical numerical values obtained above are shown below in a form in which the terms correspond to those of (18), in which $E_{2}=E_{1}, R_{2}=0$, and where $R_{f}=0$ :

$$
\begin{aligned}
R_{0} & =\frac{1}{1-0.04}(0.11+0.011) \\
& =0.126 \mathrm{ohm} .
\end{aligned}
$$

\section{A Further Circuit Modification}

There is yet another beneficial modification which may be made in the regulated power supply circuit, and which is suggested by (15) for the output voltage.

It may be noted that in (15) there is a component of output resistance due to the total effective internal resistance $R_{e}=\left(R_{e 1}+R_{e 2}+R_{e 3}\right)$ of the current-amplifying transistors $T 1, T 2$, and $T 3$, and another component due to the resistance $R_{m}$ of the ammeter. The former component is equal to $R_{e}$ divided by $G$, but the latter component is $R_{m}$ divided by $\left(R_{a} / r^{\prime}\right) G$ where $R_{a} / r^{\prime}$ is at least 100. Examination of Fig. 3 suggests that if the diode $D 2$ were connected not to the emitter of transistor $T 1$ but to the emitter of $T 2$, then the two above components of output resistance might be equal to $\left(R_{e 2}+R_{e 3}\right) / G$ and $\left(R_{m}+R_{e 1}\right) /\left(R_{a} / r^{\prime}\right) G$, respectively, and the sum of the components and hence the output resistance $R_{0}$ would be reduced.

If the above modification is introduced, analysis corresponding to that for Fig. 3 leads to

$$
\begin{aligned}
E= & {\left[n e+\frac{r}{R_{b}} n E_{2}+\frac{r^{\prime}}{R_{a}} \frac{1}{G} E_{1}+\frac{1}{G} e^{\prime}\right] } \\
& -I\left[\frac{\left(R_{e 2}+R_{e 3}\right)+R / B_{1} B_{2} B_{3}}{G}\right. \\
& \left.+\frac{R_{1}+R_{m}+R_{e 1}}{\left(R_{a} / r^{\prime}\right) G}+\frac{r}{R_{b}} n R_{2}-\frac{1+n}{n} R_{f}\right] .
\end{aligned}
$$

The result of (23) shows that the expectation described above is justified: the open-circuit output voltage $E_{0}$ is the same as before, but in the output resistance $R_{0}$, the resistance $R_{e 1}$ is now divided by $\left(R_{a} / r^{\prime}\right) G$ instead of by 


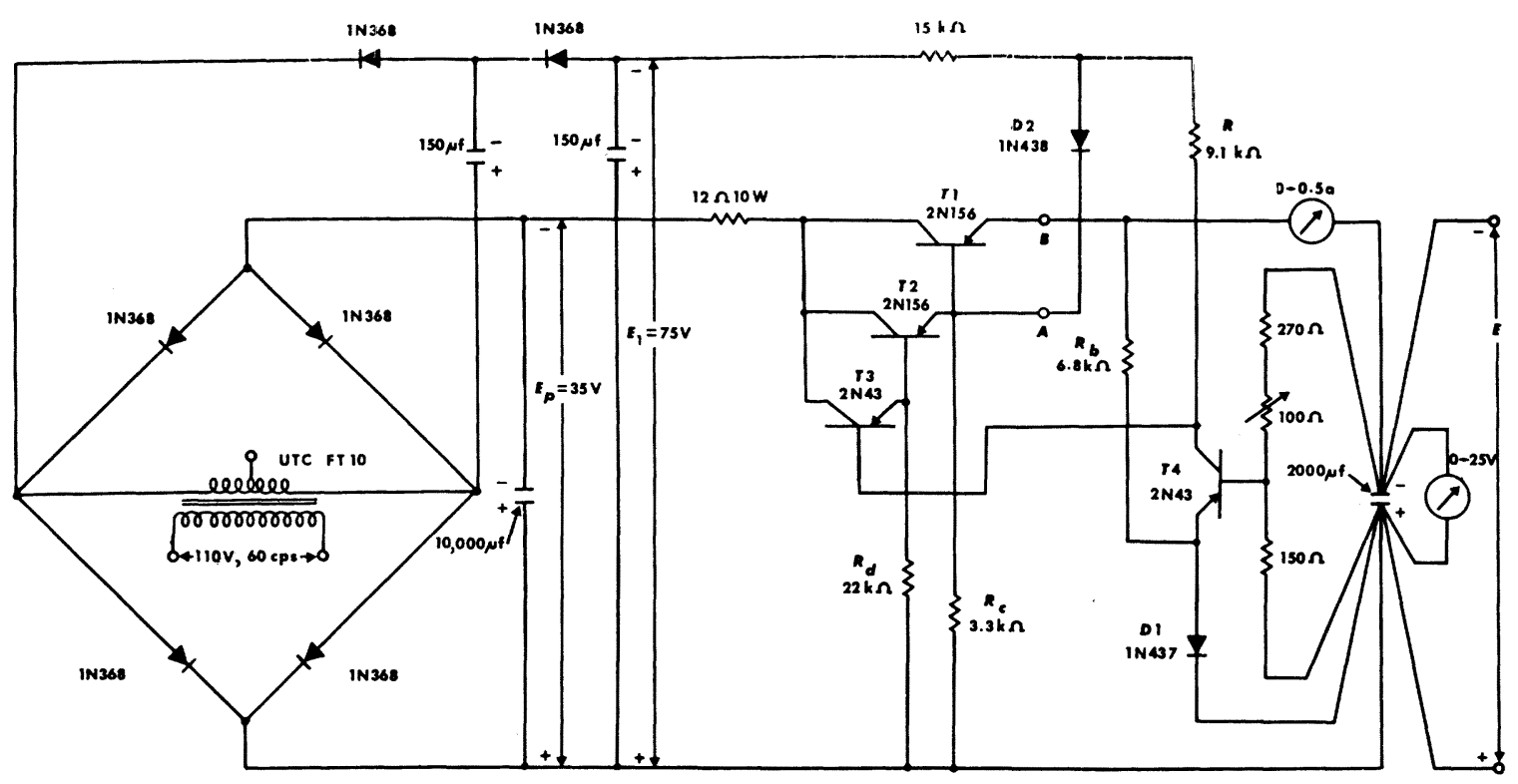

Fig. 4-Circuit of regulated supply to provide 0.5 at 18 to $22 \mathrm{v}$. The output resistance is $0.007 \mathrm{ohm}$.

$G$ alone. The same typical figures assumed before will show the improvement introduced by the circuit modification. If $G=10, R=6000 \mathrm{ohm}, B_{1}, B_{2}, B_{3}=30,40,50$, and $R_{e 1}, R_{e 2}, R_{e 3}=0.70,0.15,0.15 \mathrm{ohm}$, respectively, then

$$
\begin{aligned}
\frac{\left(R_{e 2}+R_{e 3}\right)+R / B_{1} B_{2} B_{3}}{G} & =\frac{0.3+0.1}{10} \\
& =0.04 \mathrm{ohm}
\end{aligned}
$$

and

$$
\begin{aligned}
\frac{R_{1}+R_{m}+R_{e 1}}{\left(R_{a} / r^{\prime}\right) G} & =\frac{10+1+0.7}{100 \times 10} \\
& =0.0117 \mathrm{ohm} .
\end{aligned}
$$

and if $E_{2}, R_{2}$, and $R_{f}$ are all zero the output resistance $R_{0}$ is

$$
\begin{aligned}
R_{0} & =0.04+0.0117 \\
& \approx 0.052 \mathrm{ohm}
\end{aligned}
$$

which may be compared with $R_{0}=0.121 \mathrm{ohm}$ obtained with the circuit of Fig. 3.

Obviously, whether or not the circuit modification introduces a significant reduction in $R_{0}$ depends on the actual numerical values in the circuit. For example, the improvement will not be significant if there is appreciable shunting of $R$, that is, if $R / B_{1} B_{2} B_{3}$ is comparable to $\left(R_{e 1}+R_{e 2}+R_{e 3}\right)$, or if $R_{e 1}$ is comparable with or smaller than $\left(R_{e 2}+R_{e 3}\right)$. This latter condition may occur if two transistors are used in parallel in place of the single transistor $T 1$ in order to obtain larger maximum load current $I$.

The discussion of the preceding two sections has presented the analysis and interpretation of the results to be expected from a new form of series-regulated power supply circuit. Although the treatment has been in terms of a fixed output voltage $E$, there is nothing in the principle which prevents extension to variable-voltage supplies. However, certain practical problems do arise if the output voltage is required to be varied by more than about \pm 10 per cent. In the following sections two practical power supplies embodying the above principle are described, one having an output voltage variable by \pm 10 per cent, and one having an output voltage variable over a wider range.

\section{Practical Supply No. 1: 0.5 a at 18 to $22 \mathrm{v}$}

The schematic diagram of a regulated power supply to provide up to 0.5 a at 18 to $22 \mathrm{v}$ is shown in Fig. 4 . The input power source is $110 \mathrm{v} 60 \mathrm{cps}$.

The raw supply voltage $E_{p}$ is obtained from a bridge rectifier circuit, and the auxiliary supply voltage $E_{1}$ is obtained from a voltage-doubler from the same line transformer. The auxiliary supply $E_{2}$ is not required; instead, extra bias current for the zener diode $D 2$ is obtained from the output voltage through resistor $R_{b}$, as described in Section IV.

The two resistors $R_{c}$ and $R_{d}$ are introduced so that the transistors $T 2$ and $T 3$, respectively, will not be cut off when the load current is small. The transient response is also improved because the current-amplifying transistors are working in a linear region over a greater part of their range. The presence of the resistors $R_{c}$ and $R_{d}$ modifies the details of the analysis presented in the previous sections, but the principle remains the same.

The 2000- $\mu \mathrm{f}$ capacitor connected across the regulated output is added to improve the transient response. The wiring layout indicated in Fig. 4 is particularly important: all leads should be returned directly to the capacitor terminals as shown. The reason is that the load current must not be allowed to develop undesired voltages which find their way to the amplifier input. As described in Section IV, the presence of a feedback re- 


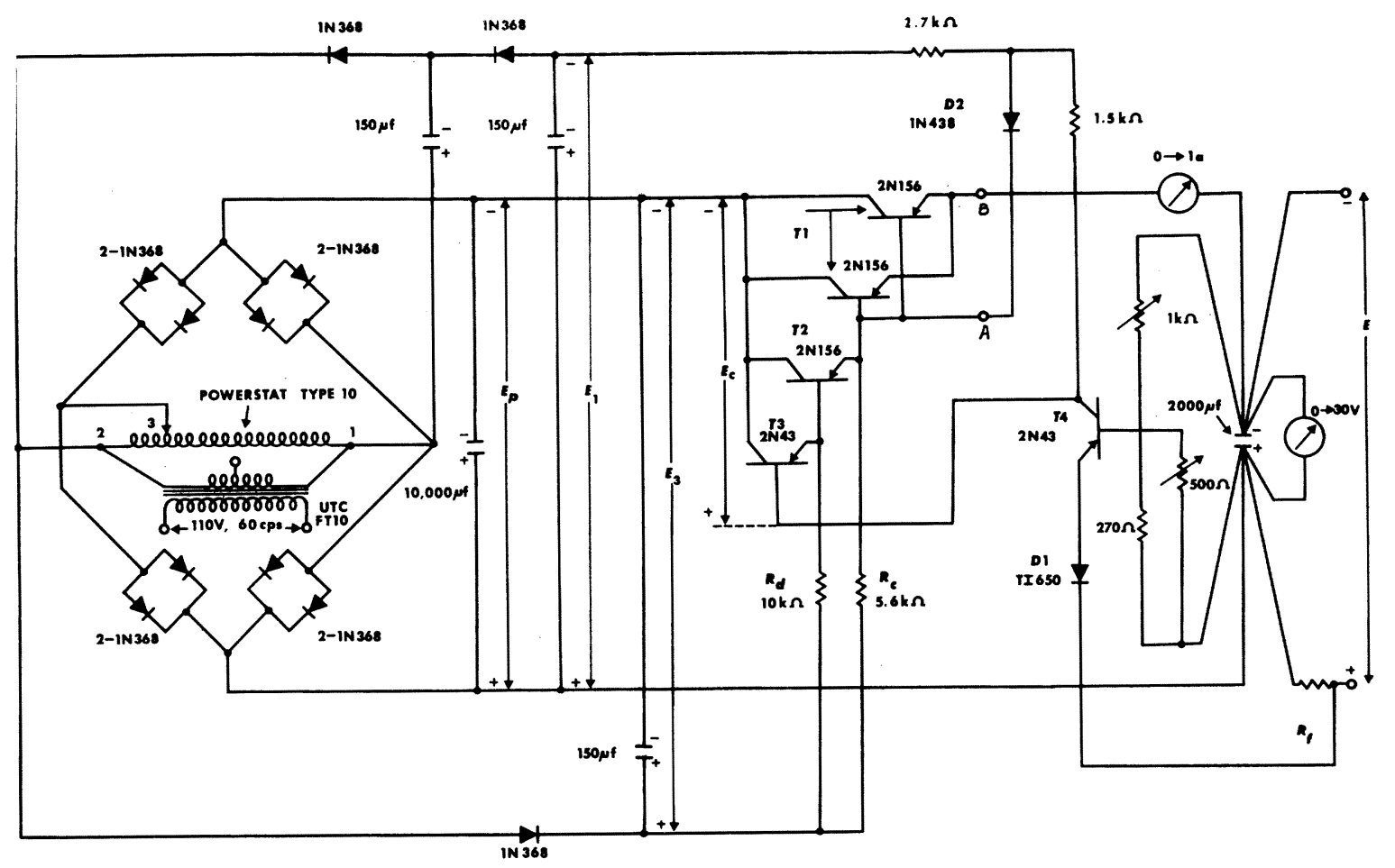

Fig. 5-Circuit of regulated supply to provide 1 a at 5 to $25 \mathrm{v}$. The output resistance can be made zero at the maximum output voltage.

sistance $R_{f}$ of only a fraction of an ohm in the right place can cause an appreciable change in output resistance.

The presence of the resistors $R_{c}$ and $R_{d}$, the difficulty in measuring the dynamic resistances of the reference diodes, and the difficulty in predicting the gain of the voltage amplifier make it rather impracticable to calculate the value of the output resistance to be expected. Experimental measurements indicate the following performance characteristics: for a suddenly applied load of $0.5 \mathrm{a}$, the output voltage drops $40 \mathrm{mv}$ and recovers in about $10 \mu \mathrm{sec}$; for a suddenly removed load of 0.5 a the output voltage rises $20 \mathrm{mv}$ and recovers in about 40 $\mu$ sec. Ripple and noise in the output are less than $5 \mathrm{mv}$ at full load, and the output voltage changes by less than $5 \mathrm{mv}$ for line voltage changes of \pm 10 per cent.

With the diode $D 2$ connected to point $\mathrm{B}$ instead of to point $A$, the steady-state output resistance is $0.03 \mathrm{ohm}$. With the diode connected to point A the output resistance is $0.007 \mathrm{ohm}$, showing that significant improvement is obtained by the circuit modification described in Section V.

\section{Practical Supply No. 2: 1 a at 5 to $25 \mathrm{v}$}

The design of a regulated power supply to provide an output voltage variable over a wide range poses several problems in addition to those already discussed for small-range supplies. The most important problem is that at low output voltage settings the power dissipation in the current-amplifying transistor becomes excessive. This can, of course, be alleviated by putting more transistors in parallel, but this is a wasteful and expensive procedure. A more attractive solution is to reduce the raw dc supply voltage $E_{p}$ by means of a Powerstat, at the same time that the output voltage $E$ is reduced, and then the power dissipation in the series transistors remains small.

The schematic of a regulated power supply to provide up to $1 \mathrm{a}$ at any voltage between 5 and $25 \mathrm{v}$ is shown in Fig. 5. The input power source is $110 \mathrm{v} 60 \mathrm{cps}$. The Powerstat is ganged to the 1 -kilohm rheostat which sets the output voltage. The regulator part of the circuit is similar in principle to the corresponding part of the $20-\mathrm{v}$ supply described in the previous section. However, the raw dc supplies in Fig. 5 are of special interest.

Three input voltages are provided: the heavy-current supply $E_{p}$, obtained from a bridge rectifier; the auxiliary supply $E_{1}$ to bias the zener diode $D 2$, obtained from a voltage doubler; and another auxiliary supply $E_{3}$ to provide current through the resistors $R_{c}$ and $R_{d}$, obtained from a half-wave rectifier. Fig. 6 shows the variation of the open-circuit characteristics of these three input voltages with Powerstat setting. The purpose of arranging the inputs in this way is to maintain all the diodes and transistors at the same operating point, and the same currents in resistors $R_{c}$ and $R_{d}$, at all settings of the output voltage.

An additional problem encountered in a power supply to provide a wide range of output voltages is that extra current for biasing zener diode $D 1$ cannot be obtained from the output voltage $E$ as was done in the $20-\mathrm{v}$ supply described previously. The reason is that since the output voltage may vary over a wide range, the extra current would vary likewise and in fact would fall to zero at the lowest output voltage. An alternative is 


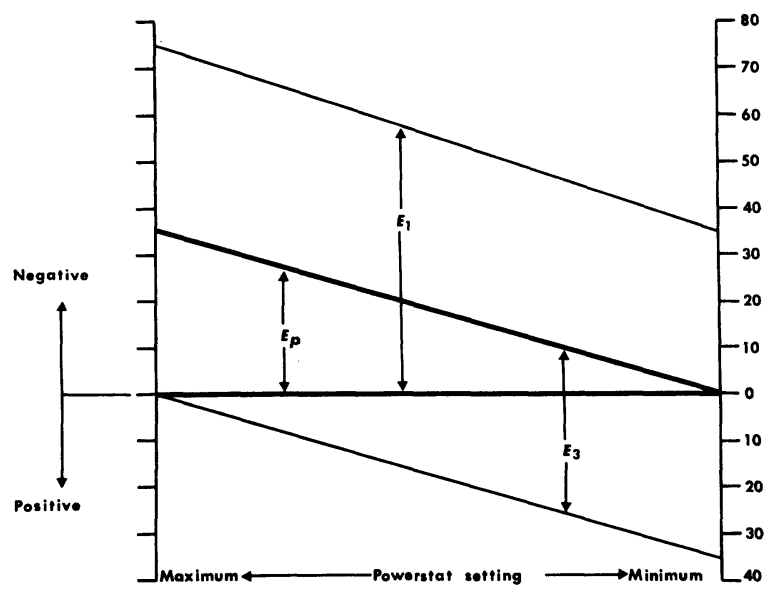

Fig. 6-Variation of the raw supply voltages, $E_{p}, E_{1}$, and $E_{3}$ with Powerstat setting, in the circuit of Fig. 5.

to derive the extra current from another auxiliary supply $E_{2}$ (as described in Section III) which does not vary with the output voltage setting. However, this is unsatisfactory because of the unavoidably large transfer internal resistance $R_{2}$ of the supply $E_{2}$, and because of the ripple introduced into the output. The only remaining possibility (short of using a second line transformer and well-filtered rectified output) is to allow sufficient current through the voltage-amplifying transistor to bias the diode $D 1$ adequately without the necessity for extra current. In the circuit of Fig. 5, the resistance $R$ is therefore made small in order to pass about $5 \mathrm{ma}$ through the voltage-amplifying transistor. Consequently, the gain of this stage is considerably less than in the supply described previously.
In the absence of the resistance $R_{f}$ in the circuit of Fig. 5, the output resistance is about $0.25 \mathrm{ohm}$ at the maximum output voltage of $25 \mathrm{v}$, irrespective of whether the diode $D 2$ is connected to point A or to point: $\mathrm{B}$. This rather large value of output resistance is principally due to the low gain of the voltage amplifier (because of the low value of $R$ ) and also to the high value of $n$ (the potential divider ratio). However, the introduction of positive current feedback as described in Section III can be usefully employed to reduce the output resistance. The circuit must be carefully wired as indicated in Fig. 5, and the resistance $R_{f}$ consists of about 30 inches of No. 22 tinned copper wire. The required length is best found by trial and error, and is chosen so that the output resistance is zero or slightly positive at maximum output voltage setting. At lower output voltages, the output resistance will then be almost zero or slightly negative. The transient response is comparable with that of the $20-\mathrm{v}$ supply described in the previous section, the actual magnitudes involved being dependent on the output voltage setting.

\section{Conclusion}

A new form of transistor series regulated power supply circuit has been introduced and analyzed. Applicacation of this new circuit to two practical regulated supplies has been described, one variable over a small range and one variable over a wide range of voltage. Each supply is simple and economical and requires only one line transformer. Independence of line voltage variations, output voltage ripple, output resistance, and transient response are unusually good.

\section{CorREGTION}

W. Guggenbuehl and M. J. O. Strutt, authors of "Theory and Experiments on Shot Noise in Semiconductor Junction Diodes and Transistors," which appeared on pages 839-854 of the June, 1957, issue of these ProceEdings, have brought the following corrections to the attention of the editors.

P. 841, (5), should read: $\overline{i_{n \text { tot }^{2}} \mid}$.

P. 843, (12), should read: $\left|\overline{i_{n} \mathrm{th}^{2}}\right|$.

P. 843, three lines below (12), should read: $\overline{i_{n I_{e}{ }^{2}} \mid}$ $=2 e I_{e} \Delta f$.

P. 843, first column, second line from bottom, should read: $\sqrt{i_{n I c}^{2}}$.

P. 843, (13), $Y$ Index should read: $Y_{L}$.

P. 845, first column, sixth line from top, should read:

$$
\left|Y_{11}\right| \gg\left|Y_{12}\right|,\left|Y_{21}\right| \gg\left|Y_{22}\right| \text {. }
$$

P. 846, first column, thirteenth line from top, should read: $\overline{\left|i_{n e}^{2}\right|}$.

P. 846, first column, fourteenth line from top, should read: $\overline{i_{n c}^{2} \mid}$.

P. 846, second column, tenth line from bottom, should read: $\overline{u_{n h}{ }^{2}}$.

P. 846, second column, ninth line from bottom, should read: $\overline{i_{n h}^{2}}$.

P. 847, first column, third line from bottom, should read:

$$
\overline{\left(i_{n 1}^{*}+i_{n 2}{ }^{*}\right)\left(i_{n 1}+i_{n s}+i_{n p}\right) .}
$$

P. 854, Acknowledgment, second line from top, should read to the firms Brown, Boveri at Baden and Hasler at Berne, Switzerland. 